\title{
Metabolomics and fluxomics in biotechnology: current trends
}

\author{
Wojciech Wojtowicz, Piotr Mlynarz * \\ Department of Bioorganic Chemistry, Wroclaw University of Technology, Wrocław, Poland
}

\begin{abstract}
Metabolomics and fluxomics are two very rapidly developing fields of science. They provide a wide range of information on different biological systems and are the last link in the biology "omics" studies chain: genomics-transcriptomics-proteomics-metabolomics. Metabolomics belongs to systems biology and it refers to changes occurring in low molecular weight compounds $(<1500 \mathrm{Da})$ and reveals significant information about the actual state of examined organisms in relation to a reference group. Metabolic flux analysis provides very important information about the flux metabolites in a pathway of a living organism based on ${ }^{13} \mathrm{C}$ enriched isotopically substrates. Both these sciences use very advanced mathematical approaches in order to gain the highest possible output originating from living systems. All features of both methods allow their use in with adopting of appropriate methodology, in a particular field of biotechnology, including medicine, pharmaceutical science and industry, food processing, toxicology, plant cultivation, and animal breeding.
\end{abstract}

Key words: metabolomics, fluxomics, biotechnology, metabolites

\section{Introduction}

Biotechnology is a field of science that utilizes living organisms or their derivatives (e.g., enzymes, natural products) in technical applications to make, modify, or process products for specific uses (Chmiel, 1994). It is applicable in broadly defined drug research, cosmetology, food industry, environment protection, and so on. The division of modern biotechnology is based on the area of application, which are assigned to a specific color that defines the field of utilization. Blue biotechnology is linked and widely used in the aquatic environment connected to marine organisms and marine renewable energy. Green biotechnology is used in agriculture to improve the biotechnological tools for crop and animal husbandry. Red biotechnology - or medical biotechnology is used in health care, in processes such as drug development, diagnostics and analysis of disease entities. White biotechnology has been adopted by the industries, and its main purpose is the creation and use of more cost - effective and better quality products. Due to the broad field of biotechnology applications, more often specialized tools are necessary for its further development (Chmiel, 1994). This is why metabolomics and fluxomics are now essential approaches in modern science.

Each of these "colors" of biotechnology is using metabolomics as a tool to obtain information about the relationship between small-molecule compounds in living organisms, their products, and needed substrates. Metabolomics approach combined with fluxomics, enable accurate tracking of changes in the distribution of low molecular weight compounds in biochemical pathways, simultaneously allowing for utilization of those relevant information in the improvement of biotechnological processes (Hou et al., 2012).

\section{Metabolomics}

Metabolomics is one of the fastest growing "omics sciences" and is a part of the systems biology. The term metabolomics was used for the first time in 2002 by $\mathrm{O}$. Fiehn. The general assumption about metabolomics is that it should enable the identification and quantification of changes occurring in the general set of metabolites

\footnotetext{
* Corresponding author: Department of Bioorganic Chemistry, Wroclaw University of Technology, Wybrzeże Wyspiańskiego 27, 50-370 Wrocław, Poland; e-mail: piotr.mlynarz@pwr.edu.pl
} 


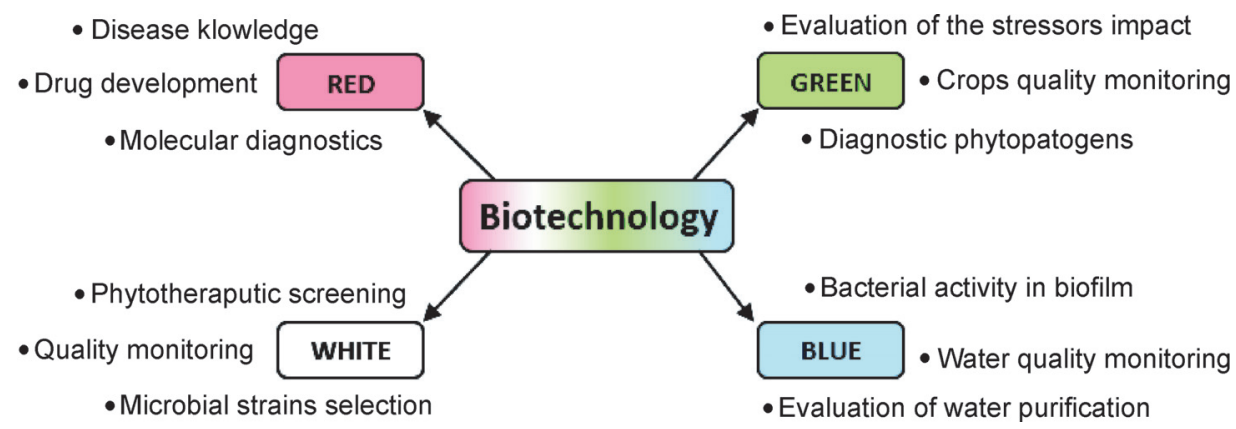

Fig. 1. An example of metabolomics and fluxomics application in biotechnology

- low molecular weight compounds (<1500 Da) - present in a tested sample (Fiehn, 2002). Due to the uniqueness of the biological material and its chemical complexity, there are some essential steps which should be performed during the process of its preparation and evaluation.

Sample storage and preparation are crucial, especially when different phases of the investigated samples (solid, liquid, and gas) are to be taken into consideration. The chosen methods should allow for obtaining the best possible metabolic "snapshot" with the minimum external influence on an overall compound composition. Another important element is the choice of an appropriate analytical technique that facilitates the broadest and the most accurate analysis of a biological matrix. The method of sample preparation and the analytical technique must ensure high reproducibility, which enables further comparative analyzes (Fiehn, 2002); therefore, special protocols were proposed in various areas of biotechnology (Vuckovic, 2012).

With the development of metabolomics, the terms metabolic fingerprint and metabolic footprint have now become commonly used. They are based on the general assumptions of metabolomics; however, the overall picture has the reduced scope to just small molecule compounds of the diverse origins. Particularly in cells type studies, metabolic fingerprinting refers directly to the whole set of intracellular metabolites (Lin et al., 2007), while metabolic footprinting applies to a set of extracellular metabolites (Pope et al., 2007).

Many analytical techniques are applied in metabolomics studies due to the vast amount of chemical compounds and their different groups. However, the most commonly used are mass spectrometry (MS) combined with a separation technique, for example, gas (GC), liquid (LC) chromatography, or capillary electrophoresis (CE) (Vuckovic, 2012), and nuclear magnetic resonance spectroscopy (NMR) with the use of different nuclei resonance frequencies (Psychogios et al., 2011). These analytical techniques allow the collection of large amounts of data from each sample, however on different "depths," considering the compounds concentration. Because the generated data matrices are extensive, there is a need to use mathematical methods, which enable dimensionality reduction and allow to visualize the obtained data. As a result of this need, the chemometric approach became widely applied in metabolomics (Holmes and Antti, 2002); however, basic statistics is also used for comparing concentrations of metabolites in different groups (Deja et al., 2013). Commonly used chemometric methods can be categorized as either supervised or unsupervised, with Principal Component Analysis (PCA), Robust Principal Component Analysis (RPCA), and Hierarchical cluster analysis (Uarrota et al., 2014) being unsupervised, and Partial least squares Discriminant Analysis (PLS-DA) (Wold et al., 2001) and Orthogonal Partial least squares Discriminant Analysis (OPLS-DA) as supervised methods.

The possibilities of analyzing, testing, and data visualization are broad and still expanding due to the growth in the field of chemometrics.

Metabolomics studies enable new and rapid diagnostic methods to be used in various cases. They are extensively used in cancer research (Deja et al., 2013; Fong et al., 2011; Hirayama et al., 2009) and might potentially support histopathological examination. These studies can be a supporting tool for final medical diagnosis, or they can become an alternative or even an independent diagnostic method (Spratlin et al., 2009). Commonly evaluated biological materials in metabolomics 

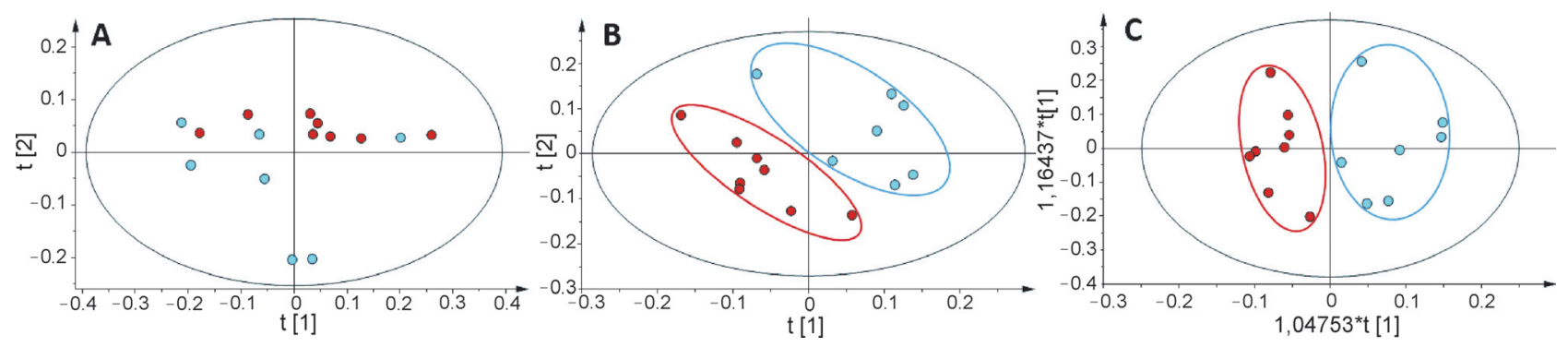

Fig. 2. Two-dimensional projection of PCA (unsupervised), PLS-DA (supervised), and OPLS-DA (supervised) chemometric models

studies are serum and urine, which very often reflects the biochemical changes occurring in organisms. Therefore, it may be an option for basic diagnostics and might enable rapid and less-invasive screening tests for patients. At the same time, metabolomics permits tracking disease changes and development or anticipating the trend of the disease's progression (Zhang et al., 2012; Dawiskiba et al., 2014; Zabek et al., 2016).

Metabolomics enabled the determination of the crops metabolome (phenotyping) by differentiating the composition of low molecular weight compounds in soybean (Lin et al., 2014), rice (Hu et al., 2014) and beans cultivars (Mensack et al., 2010), subsequently enabled the differentiation of regional products in terms of their origin (Zieliński et al., 2014) or the impact of stressors on living organisms (Dita et al., 2006). Metabolomics methods make it possible to create a distinctive set of metabolites - attributed to specific regional products which determine their originality and quality (Carlin et al., 2016). The evaluation and characterization of crops in terms of the composition of metabolites may enable quality control of final products in the food industry by changing storage conditions (Arapitsas et al., 2016) and potentially allowing to plan crops by knowing influence of environmental condition in order to achieve a higher quality by special composition of metabolome (Fernie and Schauer, 2009).

In addition to its use in the research on food industry products, metabolomics can also be used as a tool to analyze the influence of a specific diet on a living organism (Stella et al., 2006), or to provide diagnostic analysis of plants against infections present before harvest (Jahangir et al., 2008).

It is also possible to indicate the differences between genetically modified and unmodified organisms together with the influence of environmental factors on metabolome changes of a modified line with regard to the following year's crops. This approach was applied in a case study of field-grown, genetically modified wheat, in which it has been shown - based on a metabolomics analysis that the place and year of cultivation have had a significant impact on the plants' metabolome (Baker et al., 2006). The evaluation of differences in the metabolome was successfully used for differentiating wild type seeds from their biosynthetic mutants (Bottcher et al., 2008).

The metabolomics approach can also be used directly in the biotechnological industry, where microbial secondary metabolites are extremely important. It allows to track the changes of conditions in which the selected microbial strains may be more relevant than others (Hou et al., 2012). Therefore, the identification and classification of microorganisms, based on metabolome, can be very helpful. This methodology has been used with success in the taxonomic classification of fungi and yeast (Smedsgaard and Nielsen, 2005). Similarly, the designation of secondary metabolites in plants is also an important part of metabolomics studies (Orešič et al., 2006). Secondary metabolites of fungi and plants are widely applied in the biotechnological industry (Gandhi et al., 2015 ) within the use of metabolomics for the screening of biologically active compounds (Harvey, 2007) and in herbal medicine control (Zeng et al., 2007). Medication studies, due to the metabolomics approach, have gained a new dimension, allowing for continuous monitoring (fast screening) and verification of response of the tested compound aimed at the metabolome changes (Van Doorn et al., 2007). Metabolomics also found application in general toxicology as a source of information about the mechanism of toxin action or toxicity screening (Robertson et al., 2011; Bouhifd et al., 2013; Zaitsu et al., 2016). 
The general metabolomics approach can be used in quality control and natural drug development (Shyur and Yang, 2008). It can also be used in assessing the quality of a product in the food industry and in tracking the changes in composition of small molecular compounds during food processing of semi-finished products (Jawień et al., 2015). As in microorganisms and plants screening, it is possible to apply metabolomics to the process of determining the best cell line for drugs research (Tiziani et al., 2011) or fluctuations in the distribution of metabolites in various pathogenic mutations (Cuperlović-Culf et al., 2010).

Although metabolomics is able to indicate potential changes in specific biochemical pathways with the use of noticeable changes in concentration of various low molecular compounds. This could also provide important information from a biotechnological point of view. The determination of these metabolic variations with accuracy of specific metabolite flow in biochemical pathways, could be achieved by the fluxomics studies (Stephanopoulos, 1999; Crown and Antoniewicz, 2013a).

\section{Fluxomics}

Fluxomics - to be precise, ${ }^{13} \mathrm{C}$-Metabolic Flux Analysis $\left({ }^{13} \mathrm{C}-\mathrm{MFA}\right)$ - is an area of science that has been developed for about twenty years now, and it is based on the utilization of stable, isotope ${ }^{13} \mathrm{C}$-labeled substrates used for accurate tracking changes in the distribution of metabolites in biochemical pathways (Stephanopoulos, 1999; Crown and Antoniewicz, 2013a; Winter and Krömer, 2013). ${ }^{13} \mathrm{C}$-labeled glucose is often used in fluxomics studies (Metallo et al., 2009); however, it is also possible to use other labeled compounds as substrates. Various labeled compounds such as ${ }^{13} \mathrm{C}$-glutamine (Yoo et al., 2008), ${ }^{13}$ C-lactate (Bak et al., 2007), ${ }^{13} \mathrm{C}$-propionate (Burgess et al., 2004), and ${ }^{13} \mathrm{C}$-glicerol have already been used in flux studies (Jain et al., 2012).

Fluxomics studies are multistaged. In accordance with the good practice of carrying out research proposed by the Crown and Antoniewicz (Crown and Antoniewicz, 2013b), it is essential to select the right labeled compound and conduct the experiment with a proper workflow. Two main trends in ${ }^{13} \mathrm{CMFA}$ studies are single tracer experiments (Burgess et al., 2004) and parallel labeling experiments (Crown and Antoniewicz, 2013a). The choice of a particular experiment design determines the choice and the amount of different stable labeled isotope compounds that can be introduced in the process. The single tracer experiment is based on the use of a single labeled tracer or mixture for flux solving, while parallel labeling needs at least two separately used labeled compounds (Crown and Antoniewicz, 2013a). The selection of tracers for an experiment often requires optimization in order to obtain possibly the most accurate results for the metabolic pathway being the point of interest (Walther et al., 2012).

Therefore, an earlier opportunity to determine a probable location of changes in metabolism in cell lines through metabolomics research can be helpful, reducing the scope of the search for relevant tracers for a selected biochemical pathway. In fluxomics studies, ${ }^{13} \mathrm{C}$ tracer is introduced into cell cultures, and later, the biological material is gathered and analyzed in the contents of the set of "isotopically enriched ${ }^{13} \mathrm{C}$ " intracellular or extracellular metabolites (Burgess et al., 2004; Bak et al., 2007; Yoo et al., 2008; Toya et al., 2010; Jain et al., 2012).

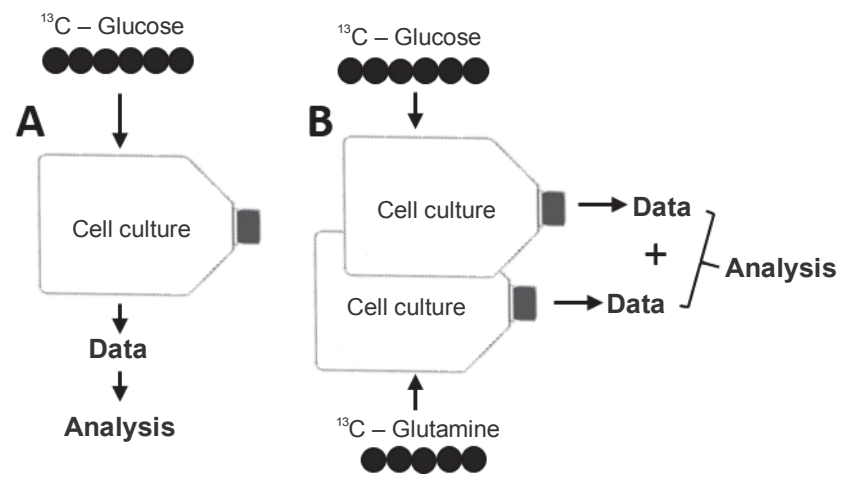

Fig. 3. Simplified scheme of differences between a Single Tracer Experiment (A) and a Parallel Labeling Experiment (B)

The next necessary step is to determine the labeled metabolites within their topoisomers and calculate their levels. Two major analytical methods are used in fluxomics - mass spectrometry (Wittmann, 2002), and nuclear magnetic resonance (Marx et al., 1996). The results obtained in the fluxomics study should, in line with the assumptions of good practice (Crown and Antoniewicz, 2013b), be tested statistically for their significance and model accuracy.

Fluxomics found its application in the basic identification of biochemical pathways research. It has been used to investigate the pathways of pentose phosphate (PP), Embden-Meyerhof-Parnas (EMP), Entner Doudoroff(ED), citric acid cycle (TCA), glycolysis and amino acids bio- 
synthesis (Blank et al., 2005; Feng et al., 2009; Tcherkez et al., 2009; Hanke et al., 2013; Klingner et al., 2015).

The utilization of ${ }^{13} \mathrm{C}-\mathrm{MFA}$ can be very broad, and due to its ability to precisely define the diversity in metabolic flows, it might be successfully applied in biotechnology.

Flux investigation allows the classification of microorganism's strains, based on the differences in metabolic flow changes (Blank et al., 2005), potentially granting a better possibility of utilization inter alia in the production of desired compounds (Chotani et al., 2000). At the same time, it gives the possibility to control and determine the best stimulating substrates to intensify the production of certain compounds (Sánchez et al., 2014), potentially allowing the increase of productivity, or to capture changes in the flow of metabolites in genetically modified microorganisms (with an increased production of amino acids) (Sánchez et al., 2014).

In addition to the common use of fluxomics in associated microorganism's studies, it can also be successfully applied to the examination of biochemical pathways in mammalian cells (Shirai et al., 2007). Flux determination of eukaryotic cells is more difficult due to the high complexity level of the entire cell culture process. Nonetheless, it is possible to track changes in biochemical reactions in case of particular compound administration for animals (Maier et al., 2009), because of which there is a very broad applicability in the field of drug development and quality control in the pharmaceutical industry.

The described examples are only a small fraction of possibilities of metabolomics and fluxomics studies, which are continuously expanding in biotechnology field.

\section{Conclusions}

Currently, metabolomics and fluxomics have become very powerful and popular tools. Their wide possibilities, developed methodology and technology help in looking positively to the future of both these "omics" sciences, regarding their utilization in biotechnology. Despite considerable similarities, each of them provides different levels of information, in terms of time and accuracy. Each study can be applied complementarily to each other or be a nominal research target. Therefore metabolomics, through its rapid and high throughput analysis of a variety of material can find application in many product analysis starting from food, cosmetic, biotechno- logical and pharmaceutical industry and finishing on medicine. Fluxomics, by a higher level of accuracy and traceability of exact changes, can also be used as an analytical tool and a source of valuable biochemical information. Continuous development of analytical techniques related to metabolomics and fluxomics reaffirms that the future for both these sciences seems encouraging. Both of them can be used as reliable sources of information in environmental, industrial, and medical biotechnology.

\section{References}

Arapitsas P., Corte A.D., Gika H., Narduzzi L., Mattivi F., Theodoridis G. (2016) Studying the effect of storage conditions on the metabolite content of red wine using HILIC LC-MS based metabolomics. Food Chemistry 197: 1331-1340.

Bak L.K., Waagepetersen H.S., Melø T.M., Schousboe A., Sonnewald U. (2007) Complex glutamate labeling from [U13Clglucose or [U-13C]lactate in co-cultures of cerebellar neurons and astrocytes. Neurochem. Res. 32(4-5): 671-680.

Baker J.M., Hawkins N.D., Ward J.L., Lovegrove A., Napier J.A., Shewry P.R., Beale M.H. (2006) A metabolomic stu$d y$ of substantial equivalence of field-grown genetically modified wheat. Plant Biotechnol. J. 4: 381-392.

Blank L.M., Kuepfer L., Sauer U. (2005) Large-scale 13C-flux analysis reveals mechanistic principles of metabolic network robustness to null mutations in yeast. Genome Biol. 6: R49.

Blank L.M., Lehmbeck F., Sauer U. (2005) Metabolic-flux and network analysis in fourteen hemiascomycetous yeasts. FEMS Yeast Res. 5: 545-58.

Böttcher C., von Roepenack-Lahaye E., Schmidt J., Schmotz C., Neumann S., Scheel D., Clemens S. (2008) Metabolome Analysis of Biosynthetic Mutants Reveals a Diversity of Metabolic Changes and Allows Identification of a Large Number of New Compounds in Arabidopsis. Plant Physiology 147: 2107-2120.

Bouhifd M., Hartung T., Hogberg H.T., Kleensang A., Zhao L. (2013) Review: Toxicometabolomics. J. Appl. Toxicol. 3: 1365-1383.

Burgess S.C., Hausler N., Merritt M., Jeffrey F.M., Storey C., Milde A., Koshy S., Lindner J., Magnuson M.A., Malloy C.R., Sherry A.D. (2004) Impaired tricarboxylic acid cycle activity in mouse livers lacking cytosolic phosphoenolpyruvate carboxykinase. J. Biol. Chem. 279: 48941-48949.

Carlin S., Vrhovsek U., Franceschi P., Lotti C., Bontempo L., Camin F., Toubiana D., Zottele F., Toller G., Fait A., Mattivi F. (2016) Regional features of northern Italian sparkling wines, identified using solid-phase micro extraction and comprehensive two-dimensional gas chromatography coupled with time-offlight mass spectrometry. Food Chem. 208: 68-80.

Chmiel A. (1994) Biotechnologia podstawy mikrobiologiczne i biochmiczne. Wyd. Nauk. PWN. 
Chotani G., Dodge T., Hsu A., Kumar M., LaDuca R., Trimbur D., Weyler W., Sanford K. (2000) The commercial production of chemicals using pathway engineering. Biochim. Biophy.s Acta 1543: 434-455.

Crown S.B., Antoniewicz M.R., (2013a) Parallel labeling experiments and metabolic flux analysis: past, present and future methodologies. Metab. Eng. 16: 21-32.

Crown S.B., Antoniewicz M.R. (2013b) Publishing 13C metabolic flux analysis studies: a review and future perspectives. Metab. Eng. 20: 42-48.

Cuperlović-Culf M., Barnett D.A., Culf A.S., Chute I. (2010) Cell culture metabolomics: applications and future directions. Drug Discov. Today 15: 610-621.

Dawiskiba T., Deja S., Mulak A., Ząbek A., Jawień E., Pawełka D., Banasik M., Mastalerz-Migas A., Balcerzak W., Kaliszewski K., Skóra J., Barć P., Korta K., Pormańczuk K., Szyber P., Litarski A., Młynarz P. (2014) Serum and urine metabolomic fingerprinting in diagnostics of inflammatory bowel diseases. World J. Gastroenterol. 20: 163-174.

Deja S., Dawiskiba T., Balcerzak W., Orczyk-Pawiłowicz M., Głód M., Pawełka D., Młynarz P. (2013) Follicular adenomas exhibit a unique metabolic profile. ${ }^{1} \mathrm{H}$ NMR studies of thyroid lesions. PLoS One. 8: e84637.

Dita M.A., Rispail N., Prats E., Rubiales D., Singh K.B. (2006) Biotechnology approaches to overcome biotic and abiotic stress constraints in legumes. Euphytica 147: 1-24.

Eriksson L., Trygg, J. (2008) Wold, S. CV-Anova for Signi-

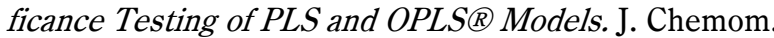
22: 594-600.

Feng X., Mouttaki H., Lin L., Huang R., Wu B., Hemme C.L., He Z., Zhang B., Hicks L.M., Xu J., Zhou J., Tang Y.J. (2009) Characterization of the central metabolic pathways in Thermoanaerobacter sp. strain X514 via isotopomer assisted metabolite analysis. Appl. Environ. Microbiol. 75: 5001-5008.

Fernie A.R., Schauer N., (2009), Metabolomics-assisted breeding: a viable option for crop improvement?, Trends Genet. 25: 39-48.

Fiehn O. (2002) Metabolomics - the link between genotypes and phenotypes. Plant Mol. Biol. 48: 155-171.

Fong M.Y., McDunn J., Kakar S.S. (2011) Identification of metabolites in the normal ovary and their transformation in primary and metastatic ovarian cancer. PLoS ONE 6: e19963.

Gandhi S.G., Mahajan V., Bedi Y.S. (2015) Changing trends in biotechnology of secondary metabolism in medicinal and aromatic plants. Planta 241: 303-317.

Hanke T., Nöh K., Noack S., Polen T., Bringer S., Sahm H., Wiechert W., Bott M. (2013) Combined fluxomics and transcriptomics analysis of glucose catabolism via a partially cyclic pentose phosphate pathway in Gluconobacter oxydans 621H. Appl. Environ Microbiol. 79: 2336-23348.

Harvey A.L. (2007) Natural products as a screening resource. Curr. Opin. Chem. Biol. 11: 480-484.

Hirayama A., Kami K., Sugimoto M., Sugawara M., Toki N., Onozuka H., Kinoshita T., Saito N., Ochiai A., Tomita M., Esumi H., Soga T. (2009) Quantitative metabolome profiling of colon and stomach cancer microenvironment by capillaryelectrophoresis time-of-flight mass spectrometry. Cancer Res. 69: 4918-4925.

Holmes E., Antti H., (2002) Chemometric contributions to the evolution of metabonomics: mathematical solutions to characterising and interpreting complex biological NMR spectra. Analyst. 127: 1549-1557.

Hou Y., Braun D.R., Michel C.R, Klassen J.L., Adnani N., Wyche T.P., Bugni T.S. (2012) Microbial strain prioritization using metabolomics tools for the discovery of natural products. Anal. Chem. 84: 4277-4283.

Hu C., Shi J., Quan S., Cui B., Kleessen S., Nikoloski Z., Tohge T., Alexander D., Guo L., Lin H., Wang J., Cui X, Rao J, Luo Q., Zhao X., Fernie A.R. Zhanget D. (2014) Metabolic variation between japonica and indica rice cultivars as revealed by non-targeted metabolomics. Sci. Rep. 4: 5067.

Jahangir M., Kim H.K., Choi Y.H., Verpoorte R. (2008) Metabolomic response of Brassica rapa submitted to pre-harvest bacterial contamination. Food Chem. 107: 362-368.

Jain M., Nilsson R., Sharma S., Madhusudhan N., Kitami T., Souza A.L., Kafri R., Kirschner M.W., Clish C.B., Mootha V.K. (2012) Metabolite profiling identifies a key role for glycine in rapid cancer cell proliferation. Science 336: 1040-1044.

Jawień E., Ząbek A., Deja S., Łukaszewicz M., Młynarz P. (2015) 1 H NMR-based metabolic profiling for evaluating poppy seed rancidity and brewing. Cell Mol. Biol. Lett. 20: 757-772.

Klingner A., Bartsch A., Dogs M., Wagner-Döbler I., Jahn D., Simon M., Brinkhoff T., Becker J., Wittmann C. (2015) Large-Scale 13C flux profiling reveals conservation of the Entner-Doudoroff pathway as a glycolytic strategy among marine bacteria that use glucose. Appl. Environ. Microbiol. 81: 2408-2422.

Lin C.Y., Wu H., Tjeerdema R.S, Viant M.R. (2007) Evaluation of metabolite extraction strategies from tissue samples using NMR metabolomics. Metabolomics 3: 55-67.

Lin H., Rao J., Shi J., Hu C., Cheng F., Wilson Z.A., Zhang D., Quan S. (2014) Seed metabolomic study reveals significant metabolite variations and correlations among different soybean cultivars. J. Integr. Plant Biol. 56: 826-836.

Maier K., Hofmann U., Bauer A., Niebel A., Vacun G., Reuss M., Mauch K. (2009) Quantification of statin effects on hepatic cholesterol synthesis by transient (13)C-flux analysis. Metab. Eng. 11: 292-309.

Marx A, de Graaf A.A., Wiechert W., Eggeling L., Sahm H. (1996) Determination of the fluxes in the central metabolism of Corynebacterium glutamicum by nuclear magnetic resonance spectroscopy combined with metabolite balancing. Biotechnol. Bioeng. 49: 111-129.

Mensack M.M., Fitzgerald V.K., Ryan E.P., Lewis M.R., Thompson H.J., Brick M.A. (2010) Evaluation of diversity among common beans (Phaseolus vulgaris L.) from two centers of domestication using 'omics' technologies. BMC Genomics 11: 686.

Metallo C.M., Walther J.L., Stephanopoulos G. (2009) Evaluation of $13 C$ isotopic tracers for metabolic flux analysis in mammalian cells. J. Biotechnol. 144: 167-174. 
Orešič M., Rischer H., Oksman-Caldentey K.M. (2006) Metabolics of plant secondary compounds: profiling of Catharanthus cell cultures. [In:] Biotechnology in Agriculture and Forestry, Vol. 57: Plant Metabolomics 277-290.

Pope G.A., MacKenzie D.A.,, Defernez M., Aroso M.A., Fuller L.J., Mellon F.A., Dunn W.B., Brown M., Goodacre R., Kell D.B., Marvin M.E., Louis E.J., Roberts I.N. (2007) Metabolic footprinting as a tool for discriminating between brewing yeasts. Yeast 24: 667-679.

Psychogios N., Hau D.D., Peng J, Guo A.C., Mandal R., Bouatra S., Sinelnikov I., Krishnamurthy R., Eisner R., Gautam B., Young N., Xia J., Knox C., Dong E., Huang P., Hollander Z., Pedersen T.L., Smith S.R., Bamforth F., Greiner R., McManus B., Newman J.W., Goodfriend T., Wishart D.S. (2011) The human serum metabolome, PloS ONE, 6: e16957.

Robertson D.G., Watkins P.B., Reily M.D. (2011) Metabolomics in toxicology: preclinical and clinical applications, Toxicol. Sci. 1: S146-170.

Sánchez C., Gómez N., Quintero J.C., Ochoa S., Rios R. (2014) A combined sensitivity and metabolic flux analysis unravel the importance of amino acid feeding strategies in clavulanic acid biosynthesis. [In:] Advances in Computational Biology, Springer International Publishing: 169-175.

Shirai T., Fujimura K., Furusawa C., Nagahisa K., Shioya S., Shimizu H. (2007) Study on roles of anaplerotic pathways in glutamate overproduction of Corynebacterium glutamicum by metabolic flux analysis. Microb. Cell Fact. 6: 19.

Shyur L.F., Yang N.S. (2008) Metabolomics for phytomedicine research and drug development. Curr. Opin. Chem. Biol. 12: 66-71.

Smedsgaard J., Nielsen J. (2005) Metabolite profiling of fungi and yeast: from phenotype to metabolome by $M S$ and informatics. J. Exp. Bot. 56: 273-286.

Spratlin J.L., Serkova N.J., Eckhardt S.G. (2009) Clinical applications of metabolomics in oncology: a review. Clin. Cancer Res. 15: 431-440.

Stella C., Beckwith-Hall B., Cloarec O., Holmes E., Lindon J.C., Powell J., van der Ouderaa F., Bingham S., Cross A.J., Nicholson J.K. (2006) Susceptibility of human metabolic phenotypes to dietary modulation. J. Proteome Res. 5: 2780-2788.

Stephanopoulos G. (1999) Metabolic fluxes and metabolic engineering. Metab. Eng. 1: 1-11.

Tcherkez G., Mahé A., Gauthier P., Mauve C., Gout E., Bligny R., Cornic G., Hodges M. (2009) In folio respiratory fluxomics revealed by $13 C$ isotopic labeling and $H / D$ isotope effects highlight the noncyclic nature of the tricarboxylic acid "cycle" in illuminated leaves. Plant Physiol. 151: 620-630.

Tiziani S., Kang Y., Choi J.S., Roberts W., Paternostro G. (2011) Metabolomic high-content nuclear magnetic resonance-based drug screening of a kinase inhibitor library. Nat. Commun. 2: 545.

Toya Y., Ishii N., Nakahigashi K., Hirasawa T., Soga T., Tomita M., Shimizu K. (2010) 13C-metabolic flux analysis for batch culture of Escherichia coli and its Pyk and Pgi gene knock- out mutants based on mass isotopomer distribution of intracellular metabolites. Biotechnol. Prog. 26: 975-992.

Triba M.N., Le Moyec L., Amathieu R., Goossens C., Bouchemal N., Nahon P., Rutledge D.N., Savarin P. (2015) PLS/OPLS models in metabolomics: the impact of permutation of dataset rows on the K-fold cross-validation quality parameters. Mol. Biosyst. 11: 13-19.

Trygg J., Wold S. (2002) Orthogonal projections to latent structures (O-PLS). J. Chemometr. 16: 119-128.

Trygg J., Lundstedt T., (2007) Chemometrics Techniques for Metabonomics. [In:] The Handbook of Metabonomics and Metabolomics, Elsevier B.V.: 171-199

Uarrota V.G., Moresco R., Coelho B., Nunes Eda C., Peruch L.A., Neubert Ede O., Rocha M., Maraschin M. (2014) Metabolomics combined with chemometric tools (PCA, $H C A, P L S-D A$ and SVM) for screening cassava (Manihot esculenta Crantz) roots during postharvest physiological deterioration. Food Chem. 161: 67-78.

van Doorn M., Vogels J., Tas A., van Hoogdalem E.J., Burggraaf J., Cohen A., van der Greef J. (2007) Evaluation of metabolite profiles as biomarkers for the pharmacological effects of thiazolidinediones in Type 2 diabetes mellitus patients and healthy volunteers. Brit. J. Clin. Pharmacol. 63: 562-574.

Vuckovic D. (2012) Current trends and challenges in sample preparation for global metabolomics using liquid chromatography-mass spectrometry. Anal. Bioanal. Chem. 403: 1523-1548.

Walther J.L., Metallo C.M., Zhang J., Stephanopoulos G. (2012) Optimization of $13 C$ isotopic tracers for metabolic flux analysis in mammalian cells. Metab. Eng. 14: 162-171.

Winter G., Krömer J.O. (2013) Fluxomics - connecting 'omics analysis and phenotypes. Environ. Microbiol. 15: 19011916.

Wittmann C. (2002) Metabolic flux analysis using mass spectrometry. Adv. Biochem. Eng. Biotechnol. 74: 39-64.

Wold S., Sjöström M., Eriksson L. (2001) PLS-regression: a basic tool of chemometrics. Chemometr. Intell. Lab. Syst. 58: 109-130.

Xia J., Broadhurst D.I., Wilson M., Wishart D.S. (2013) Translational biomarker discovery in clinical metabolomics: an introductory tutorial. Metabolomics 9: 280-299.

Yoo H., Antoniewicz M.R., Stephanopoulos G., Kelleher J.K. (2008) Quantifying reductive carboxylation flux of glutamine to lipid in a brown adipocyte cell line. J. Biol. Chem. 283: 20621-20627.

Zabek A., Swierkot J., Malak A., Zawadzka I., Deja S., BoguniaKubik K., Mlynarz P. (2016) Application of (1)H NMRbased serum metabolomic studies for monitoring female patients with rheumatoid arthritis. J. Pharm. Biomed. Anal. 17: 544-550.

Zaitsu K., Hayashi Y., Kusano M., Tsuchihashi H., Ishii A. (2016) Application of metabolomics to toxicology of drugs of abuse: a mini review of metabolomics approach to acute and chronic toxicity studies. Drug Metab. Pharmacokinet. 31: 21-26. 
Zeng Z.D., Liang Y.Z., Chau F.T., Chen S., Daniel M.K., Chan C.O. (2007) Mass spectra profiling: an effective tool for quality control of herbal medicines. Anal. Chim. Acta 604: 89-98.

Zhang L., Jin H., Guo X., Yang Z., Zhao L., Tang S., Mo P., Wu K., Nie Y., Pan Y., Fan D. (2012) Distinguishing pancreatic cancer from chronic pancreatitis and healthy indivi- duals by $1 \mathrm{H}$ nuclear magnetic resonance-based metabonomic profiles. Clin. Biochem. 13: 1064-1069.

Zieliński L., Deja S., Jasicka-Misiak I., Kafarski P. (2014) Chemometrics as a tool of origin determination of Polish monofloral and multifloral honeys. J. Agric. Food Chem. 62: 2973-2981. 